\title{
Experimental Photoionization Cross-Section Measurements in the Ground and Metastable State Threshold Region of $\mathrm{Se}^{+}$
}

\author{
N C Sterling ${ }^{1,2}$, D A Esteves ${ }^{3,4}, \mathbf{R}$ C Bilodeau ${ }^{4,5}$, A L D \\ Kilcoyne $^{4}$, E C Red ${ }^{4}, \mathbf{R}$ A Phaneuf ${ }^{3}$, and A Aguilar ${ }^{4}$ \\ ${ }^{1}$ Department of Physics and Astronomy, Michigan State University, 3248 \\ Biomedical Physical Sciences, East Lansing, MI 48824-2320, USA \\ ${ }^{2}$ NSF Astronomy and Astrophysics Postdoctoral Fellow \\ ${ }^{3}$ Department of Physics, MS 220, University of Nevada, Reno, NV 89557, USA \\ ${ }^{4}$ Advanced Light Source, Lawrence Berkeley National Laboratory, Berkeley, CA \\ 94720, USA \\ ${ }^{5}$ Department of Physics, Western Michigan University, Kalamazoo, MI 49008, \\ USA
}

\begin{abstract}
Absolute photoionization cross-section measurements are reported for $\mathrm{Se}^{+}$in the photon energy range 18.0-31.0 eV, which spans the ionization thresholds of the ${ }^{4} S_{3 / 2}$ ground state and the low-lying ${ }^{2} P_{3 / 2,1 / 2}$ and ${ }^{2} D_{5 / 2,3 / 2}$ metastable states. The measurements were performed using the Advanced Light Source synchrotron radiation facility. Strong photoexcitation-autoionization resonances due to $4 p \rightarrow n d$ transitions are seen in the cross-section spectrum and identified with a quantum-defect analysis.
\end{abstract}

PACS numbers: 32.80.Fb, 32.80.Zb, 95.30.Dr, 95.30.Ky, 97.10.Cv, 98.38.Ly

Short title: Photoionization of $\mathrm{Se}^{+}$

J. Phys. B: At. Mol. Opt. Phys: 23 May 2022 


\section{Introduction}

Photoionization is an important process in determining the ionization balance and hence the abundances of elements in astrophysical nebulae. In the last few years, it has become possible to detect neutron $(n)$-capture elements (atomic number $Z>30$ ) in a large number of ionized nebulae [1, 2]. These elements are produced by slow or rapid $n$-capture nucleosynthesis (the " $s$-process" and " $r$-process," respectively). Measuring the abundances of these elements can reveal their dominant production sites in the Universe, as well as details of stellar structure, mixing and nucleosynthesis 3 $3,4,5,6,6,7,8,9]$. These astrophysical observations provide an impetus to determine the photoionization and recombination properties of $n$-capture elements.

Various $n$-capture elements have been detected in the spectra of planetary nebulae 2, 10, 11, 12], the photoionized ejecta of evolved low- and intermediate-mass stars (1-8 solar masses). Planetary nebula progenitor stars may experience $s$-process nucleosynthesis [5, 9, 13, 14], in which case their nebulae will exhibit enhanced abundances of trans-iron elements. The level of $s$-process enrichment for individual elements is strongly sensitive to the physical conditions and mixing processes in the stellar interior [5, 7, 9].

The principal difficulty in studying $s$-process enrichments in planetary nebulae is the large uncertainties (factors of 2 to 3 ) of $n$-capture element abundances derived from the observational data. There are two root causes for these uncertainties. First, due in part to their low cosmic abundances, only one or two ions of a given $n$ capture element can be detected in individual planetary nebulae. To derive elemental abundances, corrections must be applied for the abundances of unobserved ionization stages. These corrections can be large and uncertain when the unobserved ions constitute a significant fraction of an element's overall abundance. Second, while robust ionization corrections can be derived from numerical simulations of nebulae [15, 16], this method relies on the availability of accurate atomic data for processes that affect the ionization equilibrium of each element. In photoionized nebulae, these atomic data include photoionization cross sections and rate coefficients for radiative and dielectronic recombination and charge exchange reactions. These data are unknown for the overwhelming majority of $n$-capture element ions. Uncertainties in the photoionization and recombination data of $n$-capture element ions can result in elemental abundance uncertainties of a factor of two or more [1].

The present work is part of a larger study to determine the photoionization and recombination properties of $n$-capture element ions [17, motivated by the astrophysical detection of these species and the importance of measuring their elemental abundances accurately to test theories of nucleosynthesis and stellar structure. The ultimate goal of this effort is to produce atomic data suitable for incorporation into codes that numerically simulate the thermal and ionization structure of nebulae, enabling significantly more accurate abundance determinations of trans-iron elements in astrophysical nebulae than is presently achievable. Determining these data over the range of energies and temperatures encountered in astrophysical environments necessitates a predominantly theoretical approach. However, experimental measurements are needed to constrain and establish the veracity of such calculations, particularly in the case of complex systems such as low-charge states of trans-iron elements.

Se was chosen as the first element of our investigation because it has been detected in nearly twice as many planetary nebulae as any other trans-iron element 
[2]. Experimental photoionization studies of other astrophysically observed $n$-capture elements have already been conducted by other groups for select $\mathrm{Kr}$ [18, 19] and Xe ions $[20,21]$.

This paper presents experimental determinations of the absolute $\mathrm{Se}^{+}$photoionization cross section near the ground-state ionization threshold, and is the first in a series of papers on the photoionization of low-charge Se ions (up to 5 times ionized). In Section 2, the experimental procedure for our photoionization cross-section measurements is described in detail. The results and analysis of the data are presented in Section 3, and in Section 4 we summarize our work.

\section{Experiment}

High-resolution measurements of the $\mathrm{Se}^{+}$photoionization cross section have been carried out at the Advanced Light Source (ALS) synchrotron radiation facility at Lawrence Berkeley National Laboratory in California. This experiment used the merged beams technique [22] with the Ion Photon Beamline (IPB) apparatus located at undulator beamline 10.0.1 of the ALS. A detailed description of the IPB apparatus is available in Covington et al. 23. The IPB endstation has been used for photoionization cross-section measurements of a variety of singly- and multiplycharged ions 24, 25, 26, 27, 28, 29].

Se ions were produced by gently heating solid selenium inside a resistive oven within an electron-cyclotron-resonance (ECR) ion source. These ions were accelerated to an energy of $6 \mathrm{keV}$, and a $60^{\circ}$ analyzing magnet selected $\mathrm{Se}^{+}$from the accelerated ion beam. The $\mathrm{Se}^{+}$ions were collimated with two sets of vertical and horizontal slits and focused by three electrostatic einzel lenses. The resulting collimated ion beam had a typical diameter of a few millimeters, and a current ranging from 20 to $200 \mathrm{nA}$. The ions were merged onto the axis of the counter-propagating photon beam by a pair of $90^{\circ}$ spherical bending plates. In the merged beam path, an electrical potential of $1.4 \mathrm{kV}$ was placed on the "interaction region" to energy-label photoions produced in a well-defined volume, for the purpose of absolute photoionization crosssection measurements. The interaction region consists of an isolated stainless-steel mesh cylinder with entrance and exit apertures defining an effective length of $29.4 \mathrm{~cm}$. Two-dimensional intensity distributions of the photon and ion beams were measured by commercial rotating-wire beam profile monitors installed on either side of the interaction region, and by three translating-slit scanners located within the cylinder. Downstream from the interaction region, the $\mathrm{Se}^{2+}$ product ions were separated from the parent $\mathrm{Se}^{+}$ion beam with a $45^{\circ}$ dipole demerger magnet. This directed the $\mathrm{Se}^{+}$ions to a Faraday cup, while the photoions were steered by a spherical $90^{\circ}$ electrostatic deflector onto a negatively biased stainless steel plate. The secondary electrons produced by the $\mathrm{Se}^{2+}$ collisions on this plate were recorded by a singleparticle channeltron detector. The detection efficiency has been determined on several occasions by measuring a femtoampere ion current at the stainless steel plate and comparing with the count rate generated from the channeltron. These measurements have consistently shown $100 \%$ efficiency for this detection scheme.

Photons were produced by a $10-\mathrm{cm}$ period undulator located in the $1.9 \mathrm{GeV}$ electron storage ring of the ALS. A grazing-incidence spherical grating monochromator delivered a highly collimated photon beam of spatial width less than $1 \mathrm{~mm}$ and divergence less than $0.05^{\circ}$. The photon energies were selected and scanned by rotating the grating and translating the exit slit of the monochromator, while simultaneously 
adjusting the undulator gap to maximize the beam intensity. The spectral resolution of the photon beam was controlled with the entrance and exit slits of the monochromator. The photon flux was typically $3 \times 10^{13}$ photons/sec, as measured by a silicon $\mathrm{x}$ ray photodiode (IRD, SXUV-100) that was referenced to two identical photodiodes absolutely calibrated by the National Institute of Standards and Technology (NIST) and by the National Synchrotron Light Source (NSLS). To calibrate the photon energy, the well known doubly-excited states of He [30] were measured in first, second and third order. These measurements indicated that the uncertainty in the photon energies of the reported cross sections can be conservatively estimated to be less than $10 \mathrm{meV}$. The photon beam was mechanically chopped to separate photoions from the background produced by collisions between the parent ion beam and residual gas inside the interaction region.

\section{Results and discussion}

The photoion yield for $\mathrm{Se}^{+}$was measured from $18 \mathrm{eV}$ to $31 \mathrm{eV}$ at a photon energy resolution of $28 \mathrm{meV}$ (Figures 1 and 2). The actual resolution was determined by fitting Lorentzian profiles to isolated features across the scanned energy region, and taking the average value of the fitted resonance widths. Absolute photoionization cross sections were measured at discrete photon energies with the same resolution. The photoionization spectrum was multiplied by a polynomial function to normalize the spectroscopic data to all absolute cross-section measurements, which are indicated in Figure 1 by solid circles with associated uncertainties $\$$ Absolute photoionization measurements with the IPB apparatus at the ALS typically are uncertain by $\sim 20 \%$ at a $90 \%$ confidence level [23, 31]. For a detailed discussion of uncertainty estimates for photoionization measurements with the IPB apparatus, see [23, 31]. Note that these uncertainties do not account for contamination from higher-order radiation from the undulator at low photon energies. A previous experiment on $\mathrm{Xe}^{3+} 32$ using the same beamline estimated the contamination of higher-order radiation to be $\sim 2 \%$ near $40 \mathrm{eV}$. At lower photon energies, the contamination of higher-order radiation is expected to be larger, but not by more than a factor of 2-3 compared to the contamination at $40 \mathrm{eV}$. The total experimental uncertainties of the absolute measurements are estimated to be $30 \%$, which accounts for the possible contamination of the photon beam by higherorder radiation. We note that the lowest-energy absolute cross section values at 18.5 and $21.1 \mathrm{eV}$ may not be as accurate as the others due to the effects of higher-order radiation.

Given the similarities in electronic structure of $\mathrm{Se}^{+}$and $\mathrm{O}^{+}$- both have two $p$ electrons in their outermost shell that give rise to the same ${ }^{4} S$ ground state and ${ }^{2} P$ and ${ }^{2} D$ metastable states - their photoionization spectra are expected to be similar. Indeed, a comparison between the measured $\mathrm{Se}^{+}$photoionization cross section and that of $\mathrm{O}^{+}$reported by Aguilar et al. 31] shows that to be the case. For $\mathrm{O}^{+}$, the region of the spectrum encompassing the ${ }^{2} P^{o}$ and ${ }^{4} S^{o}$ thresholds consists of very strong resonances due to $2 p \rightarrow n d$ electron excitations of the metastable ions, as well as weak resonances due to $2 p \rightarrow n s$ transitions. At our resolution of $28 \mathrm{meV}$, only the $4 p \rightarrow n d$ transitions are observed in the spectrum of $\mathrm{Se}^{+}$.

Identifications for the observed $\mathrm{Se}^{+}$structure (see Figure 2) are made based on the $\mathrm{O}^{+}$resonance identifications and the use of the quantum-defect form of the Rydberg

$\ddagger$ All uncertainties quoted in this paper are $90 \%$ confidence level estimates. 

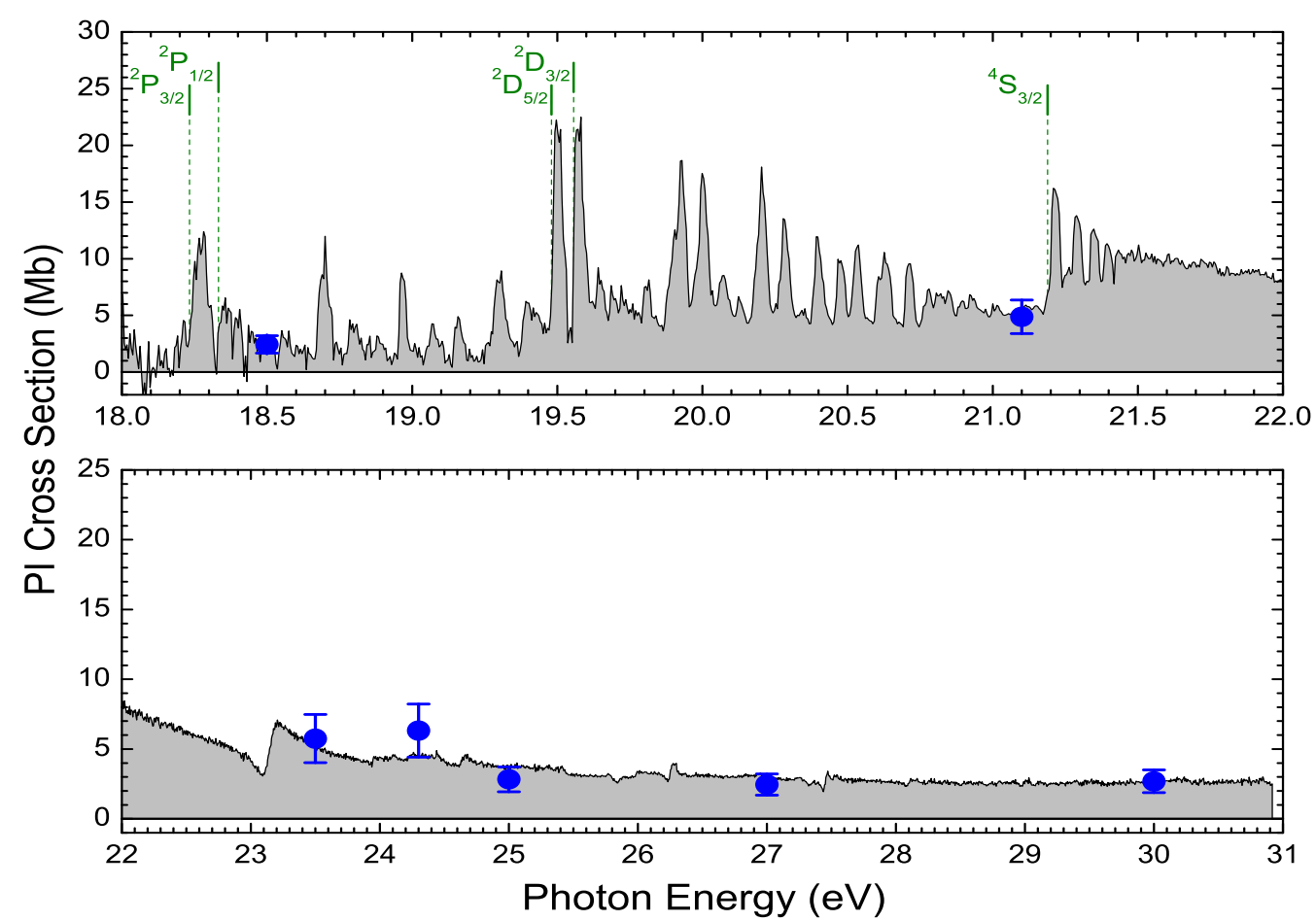

Figure 1. Absolute cross-section measurements of photoionization of $\mathrm{Se}^{+}$are shown from $18 \mathrm{eV}$ to $31 \mathrm{eV}$, at $28 \mathrm{meV}$ resolution. The ionization thresholds of the quartet ground state and the four doublet metastable states (from NIST [33]) are indicated by vertical dashed lines. The shaded region represents energy scan measurements which are normalized to absolute cross-section measurements at specific energies (solid circles with error bars).

formula,

$$
E_{n}=E_{\infty}-\frac{R(Z-N)^{2}}{\left(n-\delta_{n}\right)^{2}}
$$

where $Z$ is the charge of the nucleus, $N$ is the number of core electrons, $n$ is the principal quantum number, $E_{\infty}$ is the series limit and $\delta_{n}$ is the dimensionless quantum defect parameter that indicates the departure of the energy level $E_{n}$ from the hydrogenic value. Two series from the ${ }^{2} P_{3 / 2}^{o}$ metastable state and one from ${ }^{2} P_{1 / 2}^{o}$ have been identified. The $4 s^{2} 4 p^{2}\left({ }^{1} D\right) n d$ series converging to the ${ }^{1} D$ series limit is shown in Figure 2 by open blue triangles for resonances originating from the ${ }^{2} P_{3 / 2}^{o}$ state, and by filled blue triangles for resonances from ${ }^{2} P_{1 / 2}^{o}$. The $4 s^{2} 4 p^{2}\left({ }^{1} S\right) n d$ series converging to the ${ }^{1} S$ series limit originating from the ${ }^{2} P_{3 / 2}^{o}$ state is depicted by halffilled pink triangles in Figure 2, In addition, two other series from the ${ }^{2} D_{j}^{o}(j=5 / 2$, $3 / 2$ ) metastable states are indicated by inverted open and filled red triangles above the spectrum. These resonances correspond to the $4 s^{2} 4 p^{2}\left({ }^{1} D\right) n d$ series converging to the ${ }^{1} D$ limit. In the measured energy range, only one Rydberg series is observed from the ${ }^{4} S_{3 / 2}$ ground state, whose first autoionizing member is $4 s^{2} 4 p^{2}\left({ }^{3} P_{2}\right) 11 d$. The 
series is indicated with inverted half-open purple triangles just above the ground state threshold.

Tables 11 2 and 3 list the principal quantum numbers, resonance energies and quantum defects of the identified members of each series. The uncertainty in the quantum defect values of the first few resonances in each series is conservatively estimated to be $10 \%$. These uncertainties are a function of the energy uncertainty as well as the relative precision to which individual resonances can be identified and their associated centroids resolved. High $n$-value resonances are typically much more difficult to clearly identify as they have significantly lower intensities and are often obscured by strong low- $n$ resonances in adjacent series. High- $n$ resonances can also become unresolvable as series converge toward their respective limits. Due to these complications, uncertainties have not been estimated for high- $n$ resonances.

It is important to note that ECR ion sources are known to produce ions in the ground and metastable states in fractions that may differ from statistically-weighted values. Therefore, the reported cross-section measurements correspond to an unknown admixture of metastable and ground state fractions. In the case of $\mathrm{O}^{+} 31$ the metastable fractions were determined using the beam attenuation method $\left({ }^{4} S 43 \%\right.$, ${ }^{2} \mathrm{D} 42 \%$ and $\left.{ }^{2} P 15 \%\right)$, clearly differing from the statistically-weighted values $\left({ }^{4} S\right.$ $20 \%,{ }^{2} D 50 \%$ and ${ }^{2} P 30 \%$ ) but not as much as those reported using translational energy spectroscopy $\left({ }^{4} S 60 \%,{ }^{2} D 19 \%\right.$ and $\left.{ }^{2} P 21 \%\right)$ 34]. Similarly, we do not expect statistically-weighted metastable fractions in our $\mathrm{Se}^{+}$measurements.

\section{Summary}

The absolute photoionization cross section of $\mathrm{Se}^{+}$has been measured in the energy region of the ground state ionization threshold. The cross section exhibits a wealth of resonances that form a clear pattern of Rydberg series. The strongest resonances are identified as $4 p \rightarrow n d$ transitions belonging to the $4 s^{2} 4 p^{2}\left({ }^{1} \mathrm{D}_{2}\right) n d$ and $4 s^{2} 4 p^{2}\left({ }^{1} \mathrm{~S}_{0}\right) n d$ series originating from the ${ }^{2} P^{o}$ and ${ }^{2} D^{o}$ metastable states. The sole series from the ${ }^{4} S_{3 / 2}$ ground state is identified as $4 s^{2} 4 p^{2}\left({ }^{3} P_{2}\right) n d$. The resonance positions and quantum defects are determined for the initial members of each of these series.

The photoionization cross sections from the present study (and for other lowcharge Se ions, to be presented in forthcoming papers) will be used to calibrate a broader theoretical effort to determine the photoionization and recombination properties of astrophysically observed $n$-capture elements [17]. The resulting atomic data determinations will enable the abundances of trans-iron species in astrophysical nebulae to be derived to a much higher degree of accuracy than is currently possible, which bears implications for the nucleosynthetic sites and chemical evolution of Se and other trans-iron elements. Our absolute cross sections for $\mathrm{Se}^{+}$can be accessed via secure FTP at the IP address 131.243.76.25 (note that a username and password are required; these can be obtained by contacting A Aguilar at aaguilar@lbl.gov),or by contacting the authors N C Sterling (sterling@pa.msu.edu) and A Aguilar.

\section{Acknowledgments}

We acknowledge support by the Director, Office of Science, Office of Basic Energy Sciences, of the U.S. Department of Energy under contracts DE-AC0205CH11231, DE-AC03-76SF-00098, and grant DE-FG02-03ER15424. N C Sterling acknowledges support from an NSF Astronomy and Astrophysics Postdoctoral 


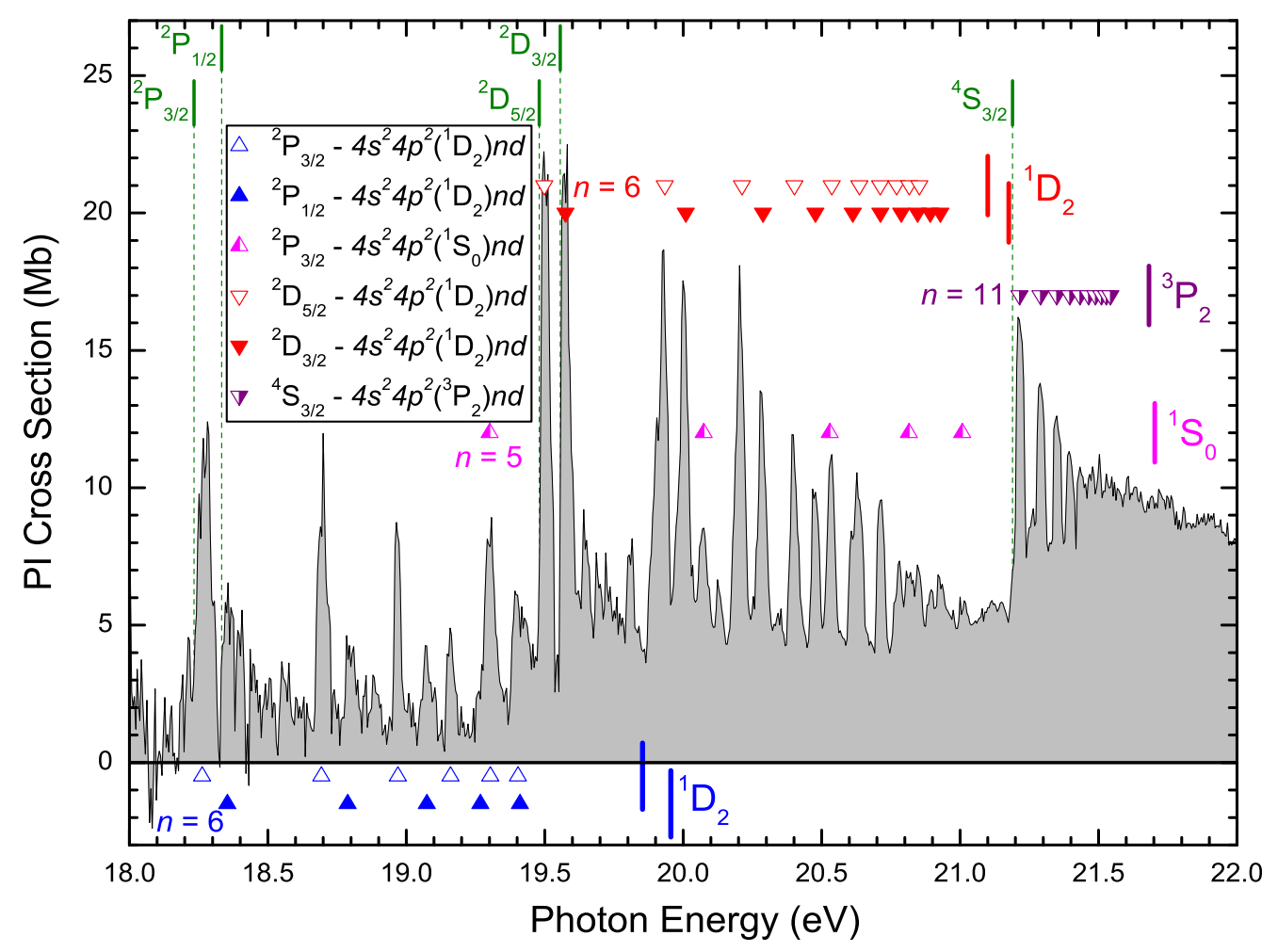

Figure 2. Absolute photoionization cross-section measurements in the region of the ground and metastable state thresholds at an energy resolution of $28 \mathrm{meV}$. Resonances originating from the ${ }^{2} P^{o}$ metastable state have been identified as members of three different Rydberg series (open and solid triangles, and half-filled pink triangles; see Table 1). Two more Rydberg series from the ${ }^{2} D^{o}$ metastable state have been distinguished (open and filled red triangles) and are listed in Table 2 All these series converge to either the ${ }^{1} D$ or the ${ }^{1} S$ excited states of $\mathrm{Se}^{2+}$ as indicated by thick vertical lines. One series originating from the ${ }^{4} S_{3 / 2}$ ground state converging to the ${ }^{3} P_{2}$ state of $\mathrm{Se}^{2+}$ has also been identified and is indicated by half-filled purple triangles. The vertical dashed lines indicate the reported metastable and ground-state ionization thresholds from NIST [33].

Fellowship under award AST-0901432 and from NASA grant 06-APRA206-0049. D. Esteves acknowledges the support from the Doctoral Fellowship Program at the Advanced Light Source. We thank Dr. Jeff Keister from Brookhaven National Laboratory and Dr. Robert Vest from NIST for performing absolute calibrations of the photodiodes.

\section{References}

[1] Sterling N C, Dinerstein H L and Kallman T R. Astrophys. J. Suppl. Ser., 169:37, 2007.

[2] Sterling N C and Dinerstein H L. Astrophys. J. Suppl. Ser., 174:157, 2008.

[3] Smith V V and Lambert D L. Astrophys. J. Suppl. Ser., 72:387, 1990.

[4] Wallerstein G et al. Rev. Mod. Phys. , 69:995, 1997. 
Table 1. Principal quantum numbers $(n)$, resonance energies and quantum defects $(\delta)$ determined from the present measurements of photoionization of $\mathrm{Se}^{+}$. Two distinct Rydberg series are observed from the ${ }^{2} P_{3 / 2}^{o}$ and one from the ${ }^{2} P_{1 / 2}^{o}$ metastable states of $\mathrm{Se}^{+}$, all due to $4 p \rightarrow n d$ transitions. The uncertainties in the experimental energies are estimated to be \pm 0.010 $\mathrm{eV}$ or less.

\begin{tabular}{|c|c|c|c|c|c|}
\hline \multicolumn{6}{|c|}{ Initial $\mathrm{Se}^{+}$state: $4 s^{2} 4 p^{3}\left({ }^{2} P_{3 / 2}^{o}\right)$} \\
\hline \multirow{2}{*}{\multicolumn{3}{|c|}{$\begin{array}{l}\text { Rydberg Series } \\
4 s^{2} 4 p^{2}\left({ }^{1} D_{2}\right) n d\end{array}$}} & \multicolumn{3}{|c|}{ Rydberg Series } \\
\hline & & & \multicolumn{3}{|c|}{$4 s^{2} 4 p^{2}\left({ }^{1} S_{0}\right) n d$} \\
\hline$n$ & Energy $(\mathrm{eV})$ & $\delta$ & $n$ & Energy $(\mathrm{eV})$ & $\delta$ \\
\hline 6 & 18.268 & 0.14 & 5 & 19.301 & 0.24 \\
\hline 7 & 18.697 & 0.14 & 6 & 20.074 & 0.22 \\
\hline 8 & 18.972 & 0.14 & 7 & 20.530 & 0.19 \\
\hline 9 & 19.160 & 0.14 & 8 & 20.815 & 0.17 \\
\hline 10 & 19.296 & 0.12 & 9 & 21.008 & 0.15 \\
\hline 11 & 19.395 & 0.1 & - & - & - \\
\hline . & . & - & . & . & \\
\hline$\infty$ & $19.853 \S$ & - & $\infty$ & $21.703 \S$ & - \\
\hline \multicolumn{6}{|c|}{ Initial $\mathrm{Se}^{+}$state: $4 s^{2} 4 p^{3}\left({ }^{2} P_{1 / 2}^{o}\right)$} \\
\hline \multirow{3}{*}{\multicolumn{6}{|c|}{$\begin{array}{l}\text { Rydberg Series } \\
4 s^{2} 4 p^{2}\left({ }^{1} D_{2}\right) n d\end{array}$}} \\
\hline & & & & & \\
\hline & & & & & \\
\hline 6 & 18.354 & 0.17 & & & \\
\hline 7 & 18.788 & 0.17 & & & \\
\hline 8 & 19.070 & 0.16 & & & \\
\hline 9 & 19.262 & 0.14 & & & \\
\hline 10 & 19.298 & 0.12 & & & \\
\hline . & . & - & & & \\
\hline$\infty$ & $19.955 \S$ & - & & & \\
\hline
\end{tabular}

$\S$ NIST tabulations 33

[5] Busso M, Gallino R and Wasserburg G J. Ann. Rev. Astron. Astrophys., 37:239, 1999.

[6] Travaglio C, Gallino R, Arnone E, Cowan J J, Jordan F and Sneden C. Astrophys. J., 601:864, 2004.

[7] Herwig F. Ann. Rev. Astron. Astrophys., 43:435, 2005.

[8] C. Sneden, J. J. Cowan, and R. Gallino. Ann. Rev. Astron. Astrophys., 46:241, 2008.

[9] Karakas A I, van Raai M A, Lugaro M, Sterling N C and Dinerstein H L. Astrophys. J., 690:1130, 2009.

[10] Péquignot D and Baluteau, J P. Astron. Astrophys, 283:593, 1994. 
Table 2. Principal quantum numbers $(n)$, resonance energies and quantum defects $(\delta)$ determined from the present measurements of photoionization of $\mathrm{Se}^{+}$. Two distinct Rydberg series are observed from the ${ }^{2} D^{o}$ metastable state of $\mathrm{Se}^{+}$, due to $4 p \rightarrow n d$ transitions. The uncertainties in the experimental energies are estimated to be $\pm 0.010 \mathrm{eV}$ or less.

\begin{tabular}{|c|c|c|c|c|c|}
\hline \multicolumn{3}{|c|}{ Initial Se state: $^{+} 4 s^{2} 4 p^{3}\left({ }^{2} D_{5 / 2}^{o}\right)$} & \multicolumn{3}{|c|}{ Initial Se state: $^{+} 4 s^{2} 4 p^{3}\left({ }^{2} D_{3 / 2}^{o}\right)$} \\
\hline \multicolumn{3}{|c|}{$\begin{array}{c}\text { Rydberg Series } \\
4 s^{2} 4 p^{2}\left({ }^{1} D_{2}\right) n d\end{array}$} & \multicolumn{3}{|c|}{$\begin{array}{c}\text { Rydberg Series } \\
4 s^{2} 4 p^{2}\left({ }^{1} D_{2}\right) n d\end{array}$} \\
\hline$n$ & Energy $(\mathrm{eV})$ & $\delta$ & $n$ & Energy $(\mathrm{eV})$ & $\delta$ \\
\hline 6 & 19.499 & 0.17 & 6 & 19.575 & 0.17 \\
\hline 7 & 19.933 & 0.17 & 7 & 20.009 & 0.17 \\
\hline 8 & 20.212 & 0.17 & 8 & 20.288 & 0.17 \\
\hline 9 & 20.402 & 0.17 & 9 & 20.478 & 0.17 \\
\hline 10 & 20.537 & 0.17 & 10 & 20.613 & 0.17 \\
\hline 11 & 20.637 & 0.16 & 11 & 20.713 & 0.16 \\
\hline 12 & 20.712 & 0.16 & 12 & 20.788 & 0.16 \\
\hline 13 & 20.770 & 0.15 & 13 & 20.846 & 0.15 \\
\hline 14 & 20.816 & 0.15 & 14 & 20.892 & 0.15 \\
\hline 15 & 20.853 & 0.15 & 15 & 20.929 & 0.15 \\
\hline . & . & - & 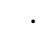 & & - \\
\hline$\infty$ & $21.100 \Perp$ & - & $\infty$ & $21.167 \|$ & - \\
\hline
\end{tabular}

|| NIST tabulations 33]

[11] Sharpee B, Zhang Y, Williams R, Pellegrini E, Cavagnolo K, Baldwin J A, Phillips M and Liu, X-W. Astrophys. J., 659:1265, 2007.

[12] Sterling N C et al. Pub. Astron. Soc. Australia, 26:339, 2009.

[13] Straniero O, Gallino R and Cristallo S. Nucl. Phys. A, 777:311, 2006.

[14] Karakas A I and Lugaro M. Pub. Astron. Soc. Australia, 27:227, 2010.

[15] Ferland G J, Korista K T, Verner D A, Ferguson J W, Kingdon J B and Verner E M. Pub. Astrophys. Soc. Pac., 110:761, 1998.

[16] Kallman T and Bautista M. Astrophys. J. Suppl. Ser., 133:221, 2001.

[17] Sterling N C and Witthoeft M C. Astron. Astrophys., in preparation.

[18] Lu M et al . Phys. Rev. A, 74:012703, 2006.

[19] Lu M, Alna'Washi G, Habibi M, Gharaibeh M F, Phaneuf R A, Kilcoyne A L D, Levenson E, Schlacter A S, Cisneros C and Hinojosa G. Phys. Rev. A, 74:062701, 2006.

[20] Bizau J M, Blancard C, Cubaynes D, Folkmann F, Champeaux J P, Lemaire J L and Wuilleumier F J. Phys. Rev. A, 73:022718, 2006.

[21] Emmons E D, Aguilar A, Gharaibeh M F, Scully S W J, Phaneuf R A, Kilcoyne A L D, Schlachter A S, Álvarez I, Cisneros C and Hinojosa G. Phys. Rev. A, 71:042704, 2005.

[22] Lyon I C, Peart B, West J B and Dolder K. J. Phys. B: At. Mol. Opt. Phys., 19:4137, 1986. 
Table 3. Principal quantum numbers $(n)$, resonance energies and quantum defects $(\delta)$ determined from the present measurements of photoionization of $\mathrm{Se}^{+}$. One distinct Rydberg series is observed from the ${ }^{4} S^{o}$ metastable state of $\mathrm{Se}^{+}$, due to $4 p \rightarrow n d$ transitions. The uncertainties in the experimental energies are estimated to be $\pm 0.010 \mathrm{eV}$ or less.

\begin{tabular}{ccc}
\multicolumn{3}{c}{ Initial Se+ ${ }^{+}$state: $4 s^{2} 4 p^{3}\left({ }^{4} S_{3 / 2}^{o}\right)$} \\
\hline \multicolumn{3}{c}{ Rydberg Series } \\
\multicolumn{3}{c}{$4 s^{2} 4 p^{2}\left({ }^{3} P_{2}\right) n d$} \\
$n$ & Energy $(\mathrm{eV})$ & $\delta$ \\
11 & 21.215 & 0.21 \\
12 & 21.291 & 0.21 \\
13 & 21.349 & 0.21 \\
14 & 21.396 & 0.21 \\
15 & 21.433 & 0.21 \\
16 & 21.464 & 0.21 \\
17 & 21.489 & 0.21 \\
18 & 21.510 & 0.21 \\
19 & 21.528 & 0.21 \\
20 & 21.543 & 0.21 \\
$\cdot$ & $\cdot$ & - \\
$\infty$ & $21.682 \Phi$ & - \\
\hline
\end{tabular}

๑ NIST tabulations 33]

[23] Covington A M et al. Phys. Rev. A., 66:062710, 2002.

[24] Müller A, Phaneuf R A, Aguilar A, Gharaibeh M F, Schlachter A S, Alvarez I, Cisneros C, Hinojosa G and McLaughlin B M. J. Phys. B: At. Mol. Opt. Phys. , 35(7):L137, 2002.

[25] Schippers S, Müller A, McLaughlin B M, Aguilar A, Cisneros C, Emmons E, Gharaibeh M F and Phaneuf R A. J. Phys. B: At. Mol. Opt. Phys. , 36:3371, 2003.

[26] Schlachter A S et al. J. Phys. B: At. Mol. Opt. Phys. , 37:L103, 2004.

[27] Scully S W J et al. J. Phys. B: At. Mol. Opt. Phys. , 38:1967, 2005.

[28] Scully S W J et al . J. Phys. B: At. Mol. Opt. Phys. , 39:3957, 2006.

[29] Müller A, Schippers S, Phaneuf R A, Kilcoyne A L D, Bräuning H, Schlachter A S, Lu M and McLaughlin B M. J. Phys. Conf. Ser., 58:383, 2007.

[30] Domke M, Schulz K, Remmers G and Kaindl G. Phys. Rev. A, 53:1424, 1996.

[31] Aguilar A et al. Astrophys. J. Suppl. Ser., 146:467, 2003.

[32] Emmons E D. Master's thesis, University of Nevada, Reno, 2004.

[33] Ralchenko Y, Kramide A E, Reader J and NIST ASD Team. National Institute of Standards and Technology, Gaithersburg, MD., 2008. http://physics.nist.gov/cgibin/AtData/main_asd3.

[34] Enos C S, Lee A R and Brenton A G. J. Phys. B: At. Mol. Opt. Phys. , 25:4021, 1992. 